\title{
Manejo quirúrgico de una fístula gastropleural posterior a manga gástrica
}

\author{
Surgical management of a gastropleural fistula after gastric sleeve \\ Henry Francisco Baptiste-Castillo', Ricardo Parra-Zuluaga² ${ }^{2}$ Freud Niño-Andrade², \\ Stefanía Rodríguez-Sánchez ${ }^{3}$
}

1 Médico, especialista en Cirugía General; coordinador, grupo de Cirujanos Generales; líder, grupo de Cirugía Bariátrica y metabólica, Clínica Amiga de Comfandi, Cali, Colombia.

2 Médico, especialista en Cirugía General, grupo de Cirugía Bariátrica y metabólica, Clínica Amiga de Comfandi, Cali, Colombia.

3 Médica, Servicio de Urgencias, Clínica Amiga de Comfandi, Cali, Colombia.

\section{Resumen}

Se presenta el caso de una paciente de 30 años de edad, sometida a un procedimiento de manga gástrica por laparoscopia en marzo de 2014, quien presenta, al tercer y cuarto años del procedimiento inicial, dos episodios de hemoptisis masiva, con falla en la segunda terapia de embolización, por lo que requirió una lobectomía pulmonar inferior izquierda. Al segundo día de este postoperatorio presenta salida de material de nutrición por las sondas de tórax, estableciéndose el diagnóstico de fistula gastro-pleural. Debido a falla con el manejo conservador, fue sometida a una resección quirúrgica de la fístula por vía laparoscópica, con gastrectomía proximal y reconstrucción en Y de Roux. A propósito de nuestra experiencia con esta paciente, se discuten en este artículo las estrategias de manejo para una fístula gastropleural, propuestas en la literatura a la fecha.

Palabras clave: cirugía bariátrica; gastrectomía; laparoscopía; procedimientos quirúrgicos mínimamente invasivos; complicaciones posoperatorias; fístula gástrica; pleura.

\begin{abstract}
This is a clinical case of a 30-year-old patient, who underwent a laparoscopic gastric sleeve procedure in March 2014, who presented, at the third and fourth years of the initial procedure, two episodes of massive hemoptysis, with failure in the second embolization therapy, requiring a lower left pulmonary lobectomy. On the second day of this postoperative period, the nutrition material came out through the chest tubes, establishing the diagnosis of gastro-pleural fistula. Due to failure of conservative management, she underwent laparoscopic surgical resection of the fistula, with proximal gastrectomy and Roux-en-Y reconstruction. Regarding our experience with this patient, this article discusses the management strategies for a gastropleural fistula, proposed in the literature to date.
\end{abstract}

Keywords: bariatric surgery; gastrectomy; laparoscopy; minimally invasive surgical procedures; postoperative complications; gastric fistula; pleura.

Fecha de recibido: 14/09/2020 - Fecha de aceptación: 02/11/2020 - Fecha de publicación en línea: 11/06/2021

Correspondencia: Henry Francisco Baptiste-Castillo, Calle 13 oeste \# 55-44, Cali, Colombia. Teléfono: 315-4372184.

Correo electrónico: baptistuta2912@gmail.com

Citar como: Baptiste-Castillo HF, Parra-Zuluaga R, Niño-Andrade F, Rodríguez-Sánchez S. Manejo quirúrgico de una fístula gastropleural posterior a manga gástrica. Rev Colomb Cir. 2021;36:712-8. https://doi.org/10.30944/20117582.771

Este es un artículo de acceso abierto bajo una Licencia Creative Commons - BY-NC-ND https://creativecommons.org/licenses/by-nc$\mathrm{nd} / 4.0 /$ deed.es 


\section{Introducción}

La progresión en el número de procedimientos de cirugía bariátrica en el mundo es significativa en los últimos 30 años, al punto que, para finales de la presente década, puede ser hasta cinco veces mayor que a principios de siglo en algunas partes del planeta, correspondiendo a la manga gástrica el mayor crecimiento ${ }^{1-3}$. De la mano con este fenómeno, se ha tornado más frecuente la presentación de complicaciones complejas. Existe por fortuna, para la mayoría de estos eventos, pautas claras de aproximación diagnóstica y tratamiento, que hacen que se logren buenos resultados, con una baja morbimortalidad ${ }^{4,5}$.

El reto se presenta por la identificación tardía de algunas complicaciones graves que, por su progresión larvada, hacen olvidar su relación con el procedimiento original, con signos y síntomas confusos que desorientan. Las alternativas de manejo son muy variadas $y$, hasta ahora, ninguna ha sido reconocida como estándar de oro.

Para el caso que nos ocupa nos referimos a la presentación y manejo de una paciente con fístula gastro-pleural compleja, que fue el hallazgo incidental luego de un procedimiento de cirugía de tórax por hemoptisis masiva recurrente, que hace pensar en que originalmente ya existía un tracto fistuloso gastro-bronquial.

\section{Caso clínico}

Paciente femenina de 30 años de edad, con antecedente de índice de masa corporal (IMC) de 39,2 $\mathrm{kg} / \mathrm{m}^{2}$ y quejas osteoarticulares en región lumbar y rodillas, que fue llevada a gastrectomía vertical por nuestro grupo Cirugía Bariátrica y metabólica, el 27 de febrero de 2014. Había egresado del programa, luego de un año de vigilancia, con IMC de $22,3 \mathrm{~kg} / \mathrm{m}^{2}$ y resolución completa de sus quejas prequirúrgicas.

Al tercer y cuarto años posoperatorios hace dos episodios de hemoptisis, manejados con embolización. Sin embargo, en la segunda oportunidad no hay control satisfactorio del sangrado, por lo que se somete en junio de 2018 a una lobectomía inferior e izquierda, orientados por una imagen diagnóstica que demostraba grave enfermedad de este lóbulo. Al segundo día postquirúrgico presenta salida de material alimentario por las sondas de tórax, lo que establece presunción diagnóstica de fístula gastro-pleural, que luego se documenta por endoscopía (Figuras 1 y 2), tomografía computarizada (Figura 3) y estudio contrastado de vías digestivas altas (Figura 4).

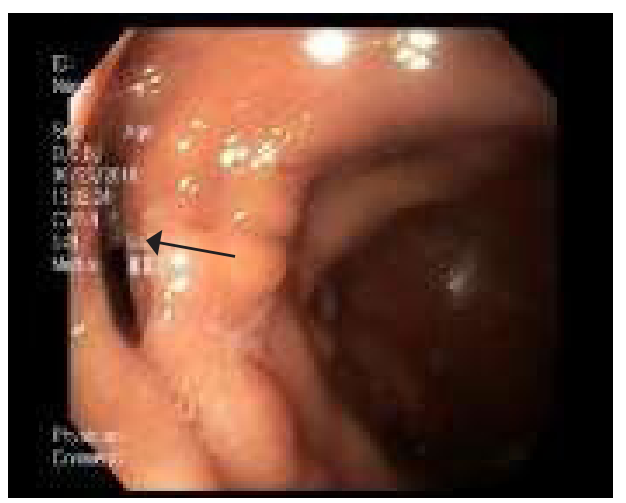

Figura 1. Gran orificio fistuloso a la izquierda (flecha).

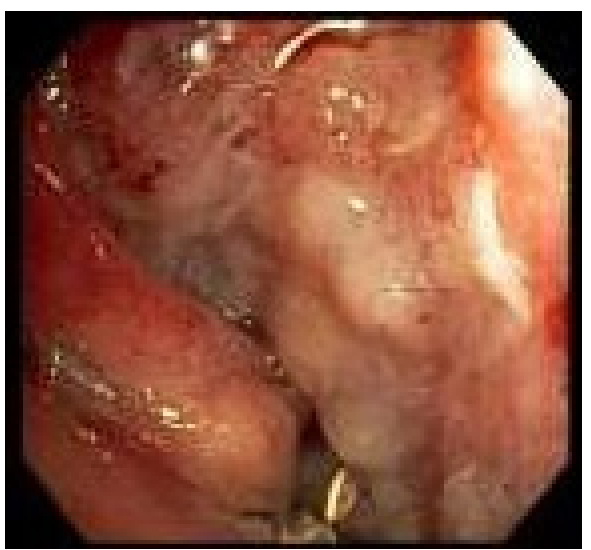

Figura 2. Cavidad de la fístula abordada con el endoscopio.

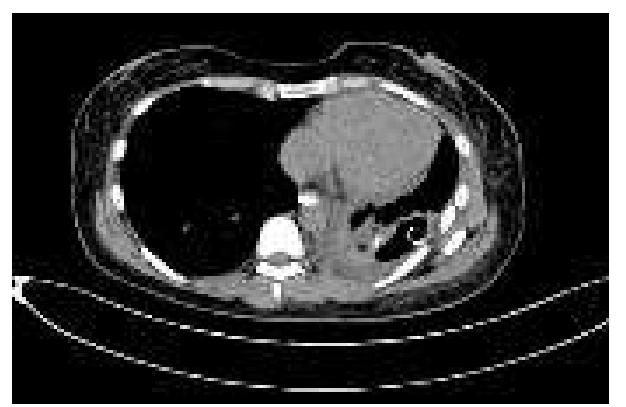

Figura 3. Tomografía computarizada donde se aprecia la colección pleural izquierda producto de la fistula. 


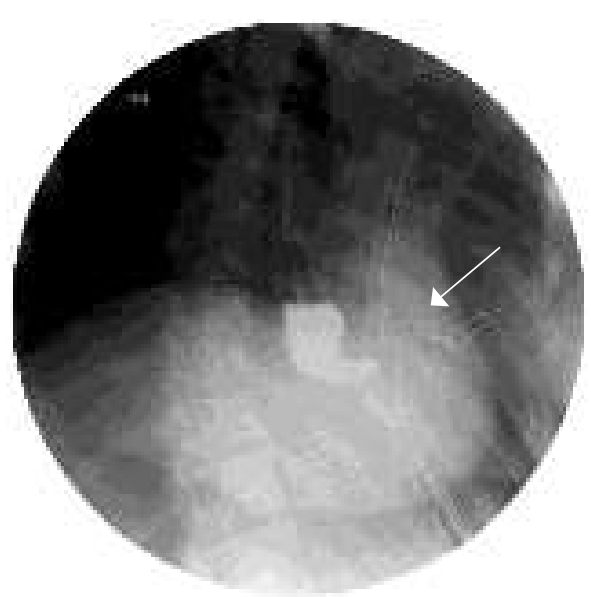

Figura 4. Se observa el medio de contraste pasando a la pleura a través de la fistula.

\section{Descripción del procedimiento quirúrgico}

En mayo de 2019, por falla en el manejo conservador, se programó nueva cirugía para la corrección quirúrgica de la fístula. Se escogió la vía laparoscópica, con los cinco puertos supraumbilicales clásicos para cirugía gastrointestinal alta que compromete el hiato. Se identificó un gran plastrón que involucraba el tercio proximal del tubo gástrico, diafragma izquierdo, polo superior del bazo, lóbulo izquierdo del hígado y el epiplón mayor.

Se liberó cada una de las estructuras involucradas en el plastrón y las adherencias del epiplón y se procedió siguiendo la antigua línea de grapado del borde gástrico a identificar la comunicación gastro-pleural, la cual se seccionó liberando el tercio proximal del estómago, lo que dejó un orificio en el diafragma de $0,5 \mathrm{~cm}$ de diámetro mayor, a $4 \mathrm{~cm}$ del pilar izquierdo, el cual se cerró con sutura no absorbible. Acto seguido se completó la gastrectomía proximal, dada la imposibilidad de sección tangencial del segmento gástrico comprometido sin producir una estenosis y por la grave isquemia del segmento proximal de la manga. Se seccionó a nivel de la zona antrocorporal del estómago y el tercio distal del esófago y se reconstruyó con una esófago- yeyunostomía en Y de Roux.

\section{Seguimiento}

Su evolución postoperatoria fue satisfactoria, lo que permitió el egreso al quinto día, tolerando la vía oral y sin colección pleural. Se documenta por controles en consulta externa hasta un año después, un curso clínico sin ninguna queja relevante, tolerando nutrición oral, con peso estable y solo discretos síntomas digestivos adaptativos secundarios a la falta de reservorio gástrico, con cierre clínico de la actividad de la fístula, cicatrización del orificio secundario en la pared del tórax y condición respiratoria sin novedades, sin hallazgos de alarma en el hemitórax izquierdo, y radiografía de tórax sin evidencia de colecciones. La endoscopia digestiva alta de control describió la anastomosis esófago-yeyunal sin soluciones de continuidad $u$ otro signo de alarma.

\section{Discusión}

Tradicionalmente se ha relacionado la presencia de fístulas derivadas de una manga gástrica a la existencia de condiciones propias del paciente, como su estado nutricional, el IMC, uso de medicamentos inmunosupresores, enfermedades crónicas que comprometen la reserva funcional y la cicatrización ${ }^{6,7}$. Otras razones tienen que ver con poco apego a una técnica depurada, escogencia inadecuada de la altura de la grapa, del diámetro de la bugía, alineación inapropiada del grapado, acercarse demasiado al ángulo de His o no alejarse racionalmente del píloro al inicio de la sutura ${ }^{8-10}$.

También se relacionan con situaciones que favorezcan el aumento de la presión intragástrica en una cirugía como la manga, que por definición produce hipertensión gástrica, tales como progresión muy rápida a sólidos, vómito recurrente y obstrucciones distales, de las cuales destacan el diseño de tubos rotados o la estenosis a la altura de la incisura angular ${ }^{6}$.

De presentación más tardía, las causas de origen vascular por enfermedades primarias que generan isquemia en las zonas críticas de vascularización, como el ángulo de His, o el fenómeno que se presenta como producto de una disección muy extensa para liberar el fondo, ya sea por falta de conciencia del riesgo o, porque forzados por la necesidad de controlar un sangrado perioperatorio significativo, se seccionan vasos críticos de la perfusión de la zona ${ }^{8,11,12}$. 
Finalmente, lo bizarro de algunos síntomas, el compromiso de sistemas diferentes al digestivo, manifestaciones no esperadas como la hemoptisis, o topografías atípicas, hacen el diagnóstico específico difícil y, por lo tanto, se retrasa el manejo definitivo, tornando la intervención quirúrgica más compleja, aun para grupos con experiencia.

La discusión se orienta para tratar de responder las preguntas usuales en este contexto. La tabla 1 resume la literatura revisada por nosotros, sobre las fístulas gastro-pleurales, tomando en cuenta en particular, aquellas publicaciones que permiten rescatar información, características y cronología.

\section{Tratamiento conservador versus tratamiento quirúrgico:}

Las posibles estrategias van desde antibióticos y nutrición, enteral o parenteral, hasta el drenaje percutáneo o quirúrgico de las colecciones, en pleura o abdomen. En la búsqueda de lograr la oclusión del orificio gástrico de la fístula, se ha recurrido al uso de pegante biológico, sutura en- doscópica, clips, o stent recubiertos; esto último de manera independiente o secuencial.

Alghanim y colaboradores describen su experiencia con el uso de stent y drenaje percutáneo de las colecciones ${ }^{14}$, y de igual manera, García y su grupo lo aplican al manejo de una fístula gastrobronquial posterior a una manga gástrica ${ }^{17}$. En su trabajo Mendoza y colaboradores, además del drenaje de las colecciones y colocación de stent recubierto en la luz del tubo gástrico, adicionan abrasión del trayecto y uso de pegante biológico ${ }^{18}$. Es importante destacar que la accesibilidad a todos estos recursos, así como la oportunidad de emplearlos a tiempo y las veces que sea necesario, no es posible en todos los escenarios.

Con relación a la utilidad del stent, se reconoce como un importante componente del manejo conservador, con buenos resultados en fistulas agudas o subagudas, pero con respuestas muy irregulares en fistulas crónicas y de la complejidad de la que nos ocupa. De la misma manera, el uso de la septotomía endoscópica es cada vez más citado como alternativa de tratamiento en fistulas recurrentes,

Tabla 1. Manejo de fistulas gastro-pleurales posteriores a manga gástrica.

\begin{tabular}{|c|c|c|c|c|}
\hline Autor & Fecha & $\begin{array}{l}\text { Tiempo de } \\
\text { latencia }\end{array}$ & Técnica & Descripción \\
\hline Boru ${ }^{13}$ & 2019 & 8 años & Manejo conservador & $\begin{array}{l}\text { Clip Ovesco, stent metálico auto- } \\
\text { expandible (SEMS), drenaje con } \\
\text { catéter pig-tail, mini baipás gástrico }\end{array}$ \\
\hline Alghanim ${ }^{14}$ & 2018 & 9 meses & Manejo conservador & Drenaje con catéter pig-tail y stent \\
\hline Andrawes ${ }^{15}$ & 2017 & 11 años & Manejo conservador & Sutura endoscópica y stent esofágico \\
\hline Nguyen ${ }^{16}$ & 2015 & 5 meses & Manejo quirúrgico & $\begin{array}{l}\text { Anastomosis esófago-yeyunostomía } \\
\text { y yeyuno-yeyunostomía, gastrostomía }\end{array}$ \\
\hline García-Quintero ${ }^{17}$ & 2015 & 11 meses & Manejo quirúrgico. & $\begin{array}{l}\text { Esofagogastrectomía con reconstruc- } \\
\text { ción en Y de Roux por laparoscopia } \\
\text { robótica }\end{array}$ \\
\hline Mendoza-Ladd 18 & 2015 & 2 meses & Manejo conservador & $\begin{array}{l}\text { Cauterización con argón plasma }+ \\
\text { fibrina humana + clip Ovesco }\end{array}$ \\
\hline lannelli ${ }^{19}$ & 2014 & 6 meses & Manejo quirúrgico & $\begin{array}{l}\text { Colocación de asa eferente en } \mathrm{Y} \text { de } \\
\text { Roux con anastomosis no muco- } \\
\text { mucosa }\end{array}$ \\
\hline Guerrero-Silva ${ }^{20}$ & 2013 & 1 mes & Manejo conservador & $\begin{array}{l}\text { Endoclip } \\
\text { Prótesis esofágica de politetrafluroe- } \\
\text { tileno y fibrina }\end{array}$ \\
\hline Serra ${ }^{21}$ & 2007 & 1 mes & Manejo conservador & $\begin{array}{l}\text { Stent auto-expandible recubierto } \\
\text { Embolización percutánea con } \\
\text { microcoil }\end{array}$ \\
\hline
\end{tabular}


pero en el caso de una fistula gastro-pleural, perpetuaría la comunicación de dos cavidades con presiones y dinámicas diferentes, y en nuestra revisión no encontramos reportes de este método para la resolución de fistulas gastro-pleurales.

El manejo operatorio propiamente dicho, involucra la resección quirúrgica de la fístula y diferentes tipos de reconstrucción de la continuidad del tracto digestivo o de un procedimiento de derivación. En lo publicado hasta la fecha en relación específicamente con este tema de las fistulas gastro-pleurales, y no de otro tipo de fístulas, la solución del problema se logra con mayor frecuencia con cirugía, ya sea como tratamiento inicial o por mala respuesta del manejo conservador, aunque los seguimientos publicados no son largos y no hay documentación del estado de los pacientes después del primer año.

\section{Tratamiento por cirugía laparoscópica versus cirugía abierta:}

En favor del abordaje laparoscópico está su accesibilidad en casi todos los medios, el progreso mundial en el uso de esta estrategia, la facilidad y menor complejidad técnica del manejo de la zona supra mesocólica y, en particular, del área en torno al hiato esofágico, cuando se aborda por esta vía ${ }^{22}$.

Al compararla con la cirugía abierta, la cirugía laparoscópica presenta una menor injuria de la pared, una más rápida recuperación de la función digestiva, menor dolor, menor estadía hospitalaria, además de un retorno más temprano a las actividades usuales. Otra ventaja probada es que, sumados los costos directos e indirectos, es una técnica menos costosa ${ }^{22,23}$

\section{Resección versus derivación:}

Cuando nos referimos a la técnica quirúrgica, es necesario reconocer que este tipo de cirugías enfrentan al cirujano, sin importar su experiencia, a situaciones inusuales y muy complejas, en las que frecuentemente se "procede según hallazgos". Siendo probable que no se pueda realizar lo planeado y que en algunas oportunidades habrá que improvisar, en lo que cabe de manera racional, siempre se debe disponer de un "plan B" con anterioridad, teniendo en mente también el escenario de retirarse sin poder hacer nada útil, para planear un segundo intento.

Es más probable, salvo que originalmente haya quedado un gran remanente de fondo, que la resección tangencial de la fístula no se pueda lograr sin una alta posibilidad de estenosis, por ello, esta es la estrategia de reparo menos viable.

Convertir a una derivación gastroyeyunal (baipás), sin resolver la fístula, es una alternativa de manejo planteado por algunos autores, con la presunción de que la reducción en las presiones del sistema, al evadir el píloro, favorece su cierre. En el caso de las fístulas gastro-pleurales, la cronicidad, la presencia de trayectos maduros y la íntima continuidad entre el tercio proximal del tubo gástrico y la pleura, hacen el tracto fistuloso muy corto y, por lo tanto, menos probable de que esta propuesta funcione ${ }^{24}$.

La resección quirúrgica de la fístula como alternativa de manejo, aunque es más compleja, tiene las ventajas de interrumpir la comunicación anormal entre los epitelios, resecar el segmento original que produce la comunicación y las infecciones recurrentes (abdominales y pleurales), y reconstruir el tránsito intestinal con tejidos sanos, con lo cual es mayor la posibilidad de retorno a sus actividades usuales mucho más temprano que con el manejo conservador, siendo más probable que esto reduzca los costos, cuando se suman los gastos directos e indirectos que cada estrategia genera $^{16,17,25}$.

\section{Gastrectomía proximal versus total:}

En favor de la gastrectomía proximal juega el hecho de una menor resección anatómica, que evita el manejo del muñón duodenal y sus riesgos, y conserva tejido del antro y parte del cuerpo para mantener un segmento de estómago funcional para la digestión. Sin embargo, no se debe desconocer el inconveniente de dejar un segmento del tracto digestivo, que puede ser asiento de patologías de todo orden, inaccesible a la evaluación y terapia endoscópica convencional, salvo con el uso de un enteroscopio. 
Tomada la decisión sobre el tipo de resección en el estómago, en lo que sí hay acuerdo es que la reconstrucción del tránsito digestivo se debe hacer con una Y de Roux ${ }^{16}$.

Una alternativa intermedia descrita por algunos autores, sin resección gástrica, dirigida específicamente a la solución quirúrgica para una fistula gastro-pleural denominada "maligna", no por su relación con neoplasias, sino porque, por sí solas son predictoras de mala respuesta al manejo medico conservador, es la liberación del fondo gástrico comprometido en la fistula y el establecimiento de una anastomosis no muco-mucosa, con un asa de yeyuno al orificio gástrico de la fístula.

Es claro que, al escoger el reparo quirúrgico para el manejo de una fístula gastro-pleural, cualquiera sea su etiología, la morbilidad y la mortalidad relacionadas son sustancialmente mayores que en la cirugía inicial, lo que hace razonable la recomendación de que sea asumida por un grupo con experiencia en las complicaciones mayores de la cirugía bariátrica, en el lugar apropiado y con la infraestructura adecuada.

\section{Conclusión}

En proporción directa al incremento de procedimientos bariátricos, como la manga gástrica por laparoscopia, aumenta también la frecuencia de complicaciones mayores, que no se limitan al postquirúrgico inmediato y mediato, sino que su presencia, como en el caso que nos ocupa, se puede hacer evidente muchos años después $y$, en oportunidades, con síntomas atípicos, por lo que es de vital importancia que siempre que un paciente con antecedente de cirugía bariátrica consulte por síntomas digestivos, dolor abdominal, quejas respiratorias o hallazgos en el tórax, se tenga en consideración que puede existir una complicación tardía de la cirugía para el manejo de la obesidad, y se proceda a buscar apoyo de quien tenga la mayor experiencia sobre el particular.

\section{Cumplimiento de normas éticas}

Consentimiento informado: Se obtuvo el consentimiento informado por parte del paciente para la publicación de las imágenes diagnósticas.
Declaración de conflicto de interés: Ninguno de los autores de este documento tiene conflicto de interés para la presentación de este caso clínico quirúrgico.

Financiación: Autofinanciado por los autores.

\section{Contribución de los autores:}

- Concepción y diseño del estudio: Henry Francisco Baptiste-Castillo.

- Adquisición de datos: Henry Francisco Baptiste-Castillo, Stefanía Rodríguez-Sánchez.

- Análisis e interpretación de datos: Henry Francisco Baptiste-Castillo, Ricardo Parra-Zuluaga, Freud NiñoAndrade, Stefanía Rodríguez-Sánchez.

- Redacción del manuscrito: Henry Francisco BaptisteCastillo, Ricardo Parra-Zuluaga, Freud Niño-Andrade, Stefanía Rodríguez-Sánchez.

- Revisión crítica: Henry Francisco Baptiste-Castillo, Ricardo Parra-Zuluaga, Freud Niño-Andrade.

\section{Referencias}

1. English WJ, DeMaria EJ, Hutter MM, Kothari SN, Mattar SG, Brethauer SA, Morton JM. American Society for Metabolic and Bariatric Surgery 2018 estimate of metabolic and bariatric procedures performed in the United States. Surg Obes Relat Dis. 2020;16:457-63. https://doi.org/10.1016/j.soard.2019.12.022

2. Buchwald H. The evolution of metabolic/bariatric surgery. Obes Surg. 2014; 24:1126-35. https://doi.org/10.1007/s11695-014-1354-3

3. Collazo-Clavell ML, Shah M. Common and rare complications of bariatric surgery. Endocrinol Metab Clin North Am. 2020;49:329-46.

https://doi.org/10.1016/j.ecl.2020.02.003

4. Peterson RM, Scott JD. Managing complications of bariatric surgery. Adv Surg. 2019;53:55-68. https://doi.org/10.1016/j.yasu.2019.04.004

5. Moon RC, Teixeira AF, Bezerra L, Alhinho HCAW, Campos J, de Quadros LG, et al. Management of bariatric complications using endoscopic stents: A multi-center study. Obes Surg. 2018;28:4034-8. https://doi.org/10.1007/s11695-018-3467-6

6. Iossa A, Abdelgawad M, Watkins BD, Silecchia G. Leaks after laparoscopic sleeve gastrectomy: Overview of pathogenesis and risk factors. Langenbecks Arch Surg. 2016;401:757-66. https://doi.org/10.1007/s00423-016-1464-6

7. Benedix F, Benedix DD, Knoll C, Weiner R, Bruns C, Manger T, et al. Are there risk factors that increase the rate of staple line leakage in patients undergoing primary sleeve gastrectomy for morbid obesity? Obes Surg. 2014;24:1610-6. https://doi.org/10.1007/s11695-014-1257-3 
8. Baker RS, Foote J, Kemmeter P, Brady R, Vroegop T, Serveld M. The science of stapling and leaks. Obes Surg. 2004;14:1290-8. https://doi.org/10.1381/0960892042583888

9. Rosenthal RJ, International Sleeve Gastrectomy Expert Panel; Diaz AA, Arvidsson D, Baker RS, Basso N, et al. International sleeve gastrectomy expert panel consensus statement: best practice guidelines based on experience of $>12,000$ cases. Surg Obes Relat Dis. 2012;8:8-19. https://doi.org/10.1016/j.soard.2011.10.019

10. Rached AA, Basile M, El Masri H. Gastric leaks post sleeve gastrectomy: Review of its prevention and management. World J Gastroenterol. 2014;20:13904-10. https://doi.org/10.3748/wjg.v20.i38.13904

11. Saber AA, Azar N, Dekal M, Abdelbaki TN. Computed tomographic scan mapping of gastric wall perfusion and clinical implications. Am J Surg. 2015;209:9991006. https://doi.org/10.1016/j.amjsurg.2014.05.023

12. Natoudi M, Theodorou D, Papalois A, Drymousis P, Alevizos L, Katsaragakis S, et al. Does tissue ischemia actually contribute to leak after sleeve gastrectomy? An experimental study. Obes Surg. 2014;24:675-83. https://doi.org/10.1007/s11695-013-1156-z

13. Boru, C.E., de Angelis, F., Iossa, A., Antypas, P., Ciccioriccio, C., Termine, P., \& Silecchia, G. (2019). Persistent fistula after sleeve gastrectomy: A chronic dilemma. Chirurgia (Romania), 114(6), 790-797. https://doi.org/10.21614/chirurgia.

14. Alghanim F, Alkhaibary A, Alzakari A, AlRumaih A. Gastropleural fistula as a rare complication of gastric sleeve surgery: A case report and comprehensive literature review. Case Rep Surg. 2018:2416915. https://doi.org/10.1155/2018/2416915

15. Andrawes S, El Douaihy Y. Using the endoscopic overstitching device and fully covered esophageal stents for closure of a gastropleural fistula and repair of a deformed gastric sleeve. VideoGIE. 2017;2:98-9. https://doi.org/10.1016/j.vgie.2017.02.003

16. Nguyen D, Dip F, Hendricks LS, Lo Menzo E, Szomstein S, Rosenthal R. The surgical management of complex fistulas after sleeve gastrectomy. Obes Surg. 2016;26:245-50.

https://doi.org/10.1007/s11695-015-1788-2
17. Garcia-Quintero P, Hernandez-Murcia C, Romero R, Derosimo J, Gonzalez A. Gastropleural fistula after bariatric surgery: a report of two cases. J Robotic Surg. 2015;9:163-6. https://doi.org/10.1007/s11701-015-0505-4

18. Mendoza-Ladd A, Al-Bayati I, Shah P, Haber G. Endoscopic closure of a gastropleural fistula. Endoscopy. 2015;47:E131-E132. https://doi.org/10.1055/s-0034-1391357

19. Iannelli A, Tavana R, Martini F, Noel P, Gugenheim J. Laparoscopic Roux limb placement over a fistula defect without mucosa-to-mucosa anastomosis: A modified technique for surgical management of chronic proximal fistulas after laparoscopic sleeve gastrectomy. Obesity Surgery. 2014;24:825-8. https://doi.org/10.1007/s11695-014-1217-y

20. Guerrero-Silva LA, López-García S, Guardado-Bermúdez F, Ardisson-Zamora FJ, Medina-Benítez A, Corona-Suárez F. Fístula gastrobronquial: complicación mayor de manga gástrica. Cirugía y Cirujanos. 2015;83:46-50. https://doi.org/10.1016/j.circir.2015.04.023

21. Serra C, Baltasar A, Andreo L, Pérez N, Bou R, Bengochea M, Chisbert JJ. Treatment of gastric leaks with coated self-expanding stents after sleeve gastrectomy. Obesity Surgery. 2007;17:866-72. https://doi.org/10.1007/s11695-007-9161-8

22. Scott-Conner CE. Laparoscopic gastrointestinal surgery. Med Clin North Am. 2002;86:1401-22. https://doi.org/10.1016/s0025-7125(02)00081-0

23. Toouli J, Cox MR. Minimal access surgery of the gastrointestinal tract. Aust N Z J Surg. 1995;65:525-32. https://doi.org/10.1111/j.1445-2197.1995.tb01799.x

24. Berry SM, Fischer JE. Classification and pathophysiology of enterocutaneous fistulas. Surg Clin North Am. 1996;76:1009-18. https://doi.org/10.1016/s0039-6109(05)70495-3

25. Al-shurafa H, Alghamdi S, Albenmousa A, Alolayan H, Al-Shurafa Z. Gastropleural fistula after single anastomosis gastric bypass. A case report and review of the literature. Inter J Surg Case Rep. 2017;35:82-6. https://doi.org/10.1016/j.ijscr.2017.03.035 